\title{
Modulation of Electrotonic Coupling in the Inferior Olive by Inhibitory and Excitatory Inputs: Integration in the Glomerulus
}

\author{
Jornt R. De Gruijl, ${ }^{1,3}$ Piotr A. Sokół, ${ }^{1,2,3}$ Mario Negrello, ${ }^{2}$ and Chris I. De Zeeuw ${ }^{1,2, *}$ \\ ${ }^{1}$ Netherlands Institute for Neuroscience, Royal Netherlands Academy of Arts \& Sciences, 1105 BA Amsterdam, the Netherlands \\ 2Department of Neuroscience, Erasmus MC, 3000 CA Rotterdam, the Netherlands \\ ${ }^{3}$ These authors contributed equally to this work \\ *Correspondence: c.dezeeuw@erasmusmc.nl \\ http://dx.doi.org/10.1016/j.neuron.2014.03.009
}

Dendritic spines in glomeruli of the inferior olive are coupled by gap junctions and receive both inhibitory and excitatory inputs. In this issue of Neuron, Lefler et al. (2014), Mathy et al. (2014), and Turecek et al. (2014) provide new insight into how these inputs modulate electrical coupling and oscillatory activity.

Whereas the intense debate between Santiago Ramón y Cajal and Camillo Golgi on the neuronal versus reticular doctrine of the CNS has been resolved more than a century ago, potential interactions between electrotonically coupled neuronal networks and afferent systems involving chemical neurotransmission have hardly been addressed. The inferior olive is one of the best nuclei to study these interactions in the mammalian brain, because it contains the highest density of neuronal gap junctions and all its coupled dendritic spines receive inhibitory synaptic input from the hindbrain and excitatory input from either ascending or descending systems (Figure 1) (De Zeeuw et al., 1998). Three papers in this issue of Neuron provide new insight into how inhibitory and excitatory inputs modulate electrical coupling in the inferior olive.

With optogenetic stimulation, Lefler et al. (2014) show that the GABAergic input from the cerebellar nuclei to the inferior olive can cause a direct but transient decrement in electrical coupling between olivary cells (Figure 1). This effect can most likely be attributed to stimulating the input to the distal, dendritic, and spiny synapses, because Lefler et al. (2014) measured, apart from a prominent impact on the coupling, a relatively consistent level of somatic postsynaptic responses to illuminations of different parts of the peripheral dendritic tree. The waveform of these postsynaptic potentials showed the characteristic frequency-dependent asynchronous release found after electri- cal stimulation of nucleo-olivary afferents (Best and Regehr, 2009). Interestingly, Lefler et al. (2014) report that the strength of the coupling between two olivary neurons is asymmetric and that the level and direction of this asymmetry can be regulated by stimulating their GABAergic input, highlighting the possibility that the spatial configuration of complex spike patterns can be created in a flexible fashion alongside its temporal aspects. This finding is in line with the asymmetric distribution of cerebellar GABAergic terminals within the mammalian olivary glomerulus (De Zeeuw et al., 1998). Thus, even though the spatial resolution of optogenetic stimulation and electrophysiological recordings has not yet reached the level of individual glomeruli, the data provided by Lefler et al. (2014) go a long way to confirm the concept of shunting coupled dendrites within glomeruli by chemical transmission (Llinás, 1974). Lefler et al. (2014) also show that activation of the GABAergic cerebellar fibers temporarily abolishes subthreshold oscillations without necessarily changing the somatic membrane potential. These data support the idea that oscillations are in part an emergent network phenomenon that depends on gap junctional current flows. This explanation is consistent with the finding that the majority of GABAergic synapses are on the dendritic spines connected by gap junctions, with far fewer synapses located directly on the dendritic shaft or soma (Sotelo et al., 1974; De Zeeuw et al., 1998). Moreover, these findings are also in line with previous work demonstrating increased doublet firing of olivary cells when GABAergic modulation of coupling within olivary glomeruli is abolished (Ruigrok et al., 1990; Van Der Giessen et al., 2008).

The reports from Mathy et al. (2014) and Turecek et al. (2014) shed new light on the effect of NMDA-dependent excitation of cells in the inferior olive. Stimulation of excitatory afferents also modifies the strength of olivary coupling, but the impact depends on calcium and CaMKII activation and is longer lasting than stimulating GABAergic afferents (i.e., tens of minutes rather than tens of milliseconds). Mathy et al. (2014) reveal a long-lasting decrease in the coupling coefficient between pairs of olivary cells subjected to $1 \mathrm{~Hz}$ electrical stimulation of adjacent white matter. In addition, Mathy et al. (2014) show that downregulating coupling between olivary cell pairs through NMDA receptor (NMDAR) activation does not depend on generation of somatic action potentials and that this activation only affects the coupling coefficient, leaving the strength of the chemical synapses unaffected. These results suggest a mechanism for long-lasting downregulation of the coupling coefficient between cells in the olive, complementing the shorter-term effect due to GABA receptor activation reported by Lefler et al. (2014). Together, these results establish that distinct mechanisms decrease the coupling between cells in the olive at different timescales (Figure 1), revealing that neural connectivity of the olive is controlled in a remarkably selective way. 


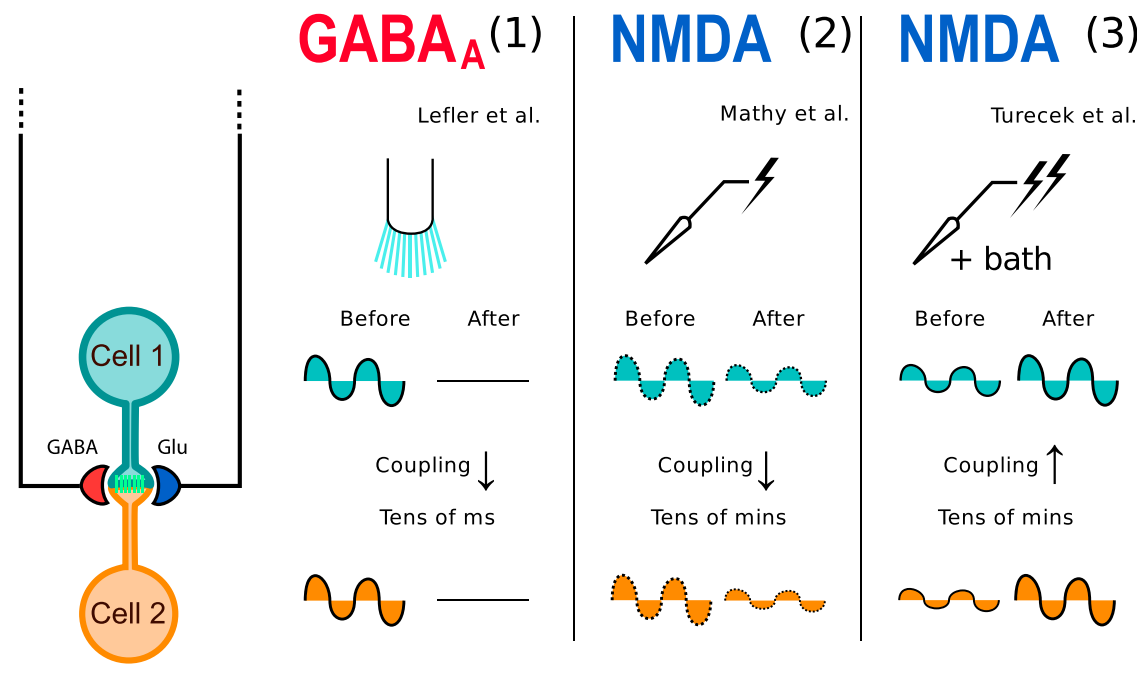

Figure 1. Modulation of Gap Junctions Reveals Multiple Forms of Plasticity

Lefler et al. (2014) (1) show that optogenetic induction of GABA release at the glomerulus creates a transient reduction of coupling coefficient; Mathy et al. (2014) (2) demonstrate that stimulation of pairs of inferior olivary cells at lower frequencies decreases the coupling coefficient; and Turecek et al. (2014) (3) reveal that stimulating olivary efferents to the glomerulus at higher frequencies increases the coupling coefficient. The waveforms in the diagrams represent the actual changes in subthreshold oscillation amplitudes as observed in (1) and (3) and the presumptive changes extrapolated from (2).

Turecek et al. (2014) also revealed a long-lasting NMDA- and CaMKII-dependent impact on the coupling coefficient between adjacent pairs of olivary cells after stimulation of their excitatory afferents. However, unlike Mathy et al. (2014), they employed stimulation at higher frequencies $(9-50 \mathrm{~Hz})$ as well as NMDA bath solutions and they observed an upregulation of coupling rather than a downregulation. Using imaging techniques, Turecek et al. (2014) demonstrate that the calcium influx remains highly local within the olivary dendrites. Combined with the unaltered amplitude of EPSPs from chemical synapses reported by Mathy et al. (2014), these findings suggest a targeted mechanism to alter the coupling coefficient between olivary neurons for longer periods of time. In fact, the two studies together offer the interesting possibility that the frequency of the excitatory input determines the strength of the coupling through the amount of calcium influx and CaMKII activation, providing a common mechanism that may allow for both upand downregulation of coupling occurring. Moreover, considering the negative impact of decoupling on oscillatory activity following stimulation of the inhibitory afferents found by Lefler et al. (2014), it is interesting to note that Turecek et al.
(2014) observed an increase in amplitude of subthreshold oscillations following stimulation of the excitatory afferents, highlighting the possibility of integrated regulation of coupling and oscillations by olivary afferents.

Several open questions remain to be addressed. Did the different postnatal ages of the animals used in the different studies (varying from postnatal day 18 to 180) have impact on the outcome of the experiments? To what extent do glomerular and extraglomerular (i.e., dendritic) GABAergic terminals serve different functions? Can a common calcium-dependent mechanism indeed explain the differential effects of stimulating excitatory inputs at different frequencies? With respect to the latter two questions, we asked whether the data from the three current studies on the olive can be unified by an extended version of a previously published multicompartmental model (De Gruijl et al., 2012). Our preliminary simulations suggest that a combination of synaptic activation on the main dendrite and at the glomeruli could account for the postsynaptic potentials recorded by Lefler et al. (2014), while activation of synapses on the main dendrite resulted in a synaptic potential resembling those they reported as a somatic voltage response. In our sim- ulations, we found that the addition of concurrent glomerular synapse stimulation resulted in a depolarizing shift in the steady-state shunt potential. Summation of the synaptic potential depended critically on the relative conductances and potentially explains the reported abolition of oscillations without noticeable somatic hyperpolarization. While glomerular and dendritic synapses may not be functionally distinct ensembles, any dendritic shunt disrupts the dendrosomatic current flow critical for generating oscillations and, therefore, stimulating only glomerular synapses as well as stimulating both glomerular and dendritic synapses concurrently stops oscillations even in intrinsically oscillating cells.

As for the findings of Mathy et al. (2014) and Turecek et al. (2014), we asked whether a common calcium-dependent mechanism can explain the bidirectional regulation of electrical coupling strength upon stimulation of the NMDA-driven excitatory inputs at different frequencies. As before, we used an extended version of the model published in De Gruijl et al. (2012) and estimated the amount of intracellular calcium in the spine head for gap junction plasticity induction protocols from both labs (without taking internal stores into account) and fitted a plasticity function (O'Donnell et al., 2011) to those results. The resulting fit was sign consistent and could qualitatively explain both labs' reported findings. Additionally, our simulations and the observations of Turecek et al. (2014) suggest that the majority of cells are damped oscillators, which require external input to oscillate (e.g., from coupled intrinsic oscillators), a finding that is in line with the hypothesis that oscillations are a network effect and spread via gap junctional coupling (Torben-Nielsen et al., 2012; Lefler et al., 2014).

In the future, we will need to gain a deeper understanding of the interplay between GABA and NMDA receptor activity patterns, thereby elucidating the function and timing properties of projections to the inferior olive. We also need to clarify to what extent the regulation of coupling in the olivocerebellar system plays a role in both acute motor performance and long-term motor learning processes (Van Der Giessen et al., 2008). The network state of the inferior olive 
determines the number of sodium spikes fired per event by individual cells (Maruta et al., 2007; Mathy et al., 2009) and may thereby mediate the direction and speed of learning in Purkinje cells (Mathy et al., 2009; Rasmussen et al., 2013). The phase of subthreshold oscillations in the inferior olive could be a determining factor for guiding climbing fiber-induced plasticity (Mathy et al., 2009; De Gruijl et al., 2012), indicating a possible role for a GABAergic reset of olivary oscillations in both motor timing and learning. In addition, inferior olive ensemble oscillation synchrony may determine the speed and direction of cerebellar learning (Bazzigaluppi et al., 2012; De Gruijl et al., 2012), which would emphasize the importance of correct segregation of inferior olive ensembles by GABAergic input from the cerebellum. As a result, cerebellar motor execution and motor learning hypotheses are now increasingly finding common ground. Spatiotemporal firing patterns of the olivocerebellum affect both motor execution and plasticity, and plasticity effects take place throughout the olivocer- ebellar system, apparently even down to the level of electrical synapses of the inferior olive (Lefler et al., 2014; Mathy et al., 2014; Turecek et al., 2014). We may not know the exact inner workings of the olivocerebellar system yet, let alone that of other loci in the CNS with chemicalelectrical interacting synapses, but work done in the labs of Yarom, Häusser, and Welsh shows that we move toward that goal with leaps and bounds.

\section{REFERENCES}

Bazzigaluppi, P., De Gruijl, J.R., Van Der Giessen, R.S., Khosrovani, S., De Zeeuw, C.I., and De Jeu, M.T.G. (2012). Front. Neural Circuits 6, 91.

Best, A.R., and Regehr, W.G. (2009). Neuron 62, 555-565.

De Gruijl, J.R., Bazzigaluppi, P., de Jeu, M.T.G., and De Zeeuw, C.I. (2012). PLoS Comput. Biol. 8, e1002814.

De Zeeuw, C.I., Simpson, J.I., Hoogenraad, C.C. Galjart, N., Koekkoek, S.K.E., and Ruigrok, T.J.H. (1998). Trends Neurosci. 21, 391-400.

Lefler, Y., Yarom, Y., and Uusisaari, M. (2014). Neuron 81 , this issue, 1389-1400.
Llinás, R. (1974). Physiologist 17, 19-46.

Maruta, J., Hensbroek, R.A., and Simpson, J.I. (2007). J. Neurosci. 27, 11263-11270.

Mathy, A., Ho, S.S.N., Davie, J.T., Duguid, I.C., Clark, B.A., and Häusser, M. (2009). Neuron 62, 388-399.

Mathy, A., Clark, B., and Häusser, M. (2014) Neuron 81, this issue, 1290-1296.

O'Donnell, C., Nolan, M.F., and van Rossum, M.C.W. (2011). J. Neurosci. 31, 16142-16156.

Rasmussen, A., Jirenhed, D.-A., Zucca, R., Johansson, F., Svensson, P., and Hesslow, G. (2013). J. Neurosci. 33, 13436-13440.

Ruigrok, T.J., de Zeeuw, C.I., and Voogd, J. (1990). Eur. J. Morphol. 28, 224-239.

Sotelo, C., Llinas, R., and Baker, R. (1974). J. Neurophysiol. 37, 541-559.

Torben-Nielsen, B., Segev, I., and Yarom, Y. (2012). PLoS Comput. Biol. 8, e1002580.

Turecek, J., Yuen, G.S., Han, V.Z., Zeng, X.-H., Bayer, K.U., and Welsh, J. (2014). Neuron 81, this issue, 1375-1388.

Van Der Giessen, R.S., Koekkoek, S.K., van Dorp, S., De Gruijl, J.R., Cupido, A., Khosrovani, S., Dortland, B., Wellershaus, K., Degen, J., Deuchars, J., et al. (2008). Neuron 58, 599-612.

\title{
Active Dendrites under Parental Supervision
}

\author{
Thomas G. Oertner ${ }^{1, *}$ \\ ${ }^{1}$ Institute for Synaptic Physiology, Center for Molecular Neurobiology Hamburg, Falkenried 94, 20251 Hamburg, Germany \\ *Correspondence: thomas.oertner@zmnh.uni-hamburg.de \\ http://dx.doi.org/10.1016/j.neuron.2014.02.036
}

Pyramidal cell dendrites are able to produce a variety of active calcium signals in brain slices. In this issue of Neuron, Grienberger et al. (2014) investigate dendritic function in the hippocampus of live mice.

Fluorescent $\mathrm{Ca}^{2+}$ indicators have forever changed our view of how neurons work. Rather than passively propagating synaptic currents to the soma, a rich repertoire of active events has been discovered in pyramidal cell dendrites, including $\mathrm{Na}^{+}$ spikes, $\mathrm{Ca}^{2+}$ spikes, NMDA spikes, and wave-like $\mathrm{Ca}^{2+}$ release events from intracellular $\mathrm{Ca}^{2+}$ stores (Schiller et al., 2000; Nakamura et al., 1999). In the past, most calcium imaging studies have been conducted in brain slices, and some forms of dendritic calcium signaling can be observed only under quite specific stimulation conditions. Clearly, the spatial distribution of excitatory inputs, the degree and timing of inhibition, and the presence or absence of modulatory inputs all affect the frequency and extent of dendritic calcium signals.

In this issue of Neuron, Grienberger et al. (2014) investigate dendritic $\mathrm{Ca}^{2+}$ signals in hippocampal pyramidal cells of live mice. As even two-photon micro- scopy cannot penetrate brain tissue deeper than about $1 \mathrm{~mm}$, the authors removed a small portion of neocortex to gain optical access to the hippocampus. Individual pyramidal cells in CA1 were loaded with the high-affinity calcium dye OGB1 through a patch pipette. Two types of calcium signals occurred spontaneously in anesthetized mice, reflecting ongoing physiological activity: very small and localized calcium "blips" were associated with small somatic 